\title{
Late Rise Human Chorionic Gonadotropin after Embryo Transfer: Causality and Significance! A mini review
}

\author{
Mohammad Jaffa ${ }^{1}$, Layal Hamdar ${ }^{2}$, Anastasia Salame ${ }^{3 *}$ \\ ${ }^{1}$ Department of Obstetrics and Gynecology, American University of Beirut Medical Center, Beirut-Lebanon \\ ${ }^{2}$ Department of Obstetrics and Gynecology, American University of Beirut Medical Center, Beirut- Lebanon \\ ${ }^{3}$ Reproductive Endocrinology and Infertility, Fakih Medical Center, Al Ain-UAE
}

*Corresponding author: Anastasia Salame, Reproductive Endocrinology and Infertility, Fakih Medical Center, Al Ain-UAE; Phone number: +971543996368; Email: anastasia.salame@fakihivf.com

Received: June 12, 2019; Accepted: June 24, 2019; Published: July 09, 2019;

\begin{abstract}
The reliable detection of hCG in maternal blood usually coincides with the embryonic implantation phase which is around 7 days post fertilization. It is believed that hCG levels taken post embryo transfers have a diagnostic and prognostic value when it comes to reproductive outcomes. Very low initial hCG levels predict a higher risk of chemical pregnancies, miscarriages and ectopic pregnancies. A review of the literature was performed so as to understand the mechanisms leading to late hCG rises as well as significance of such findings.
\end{abstract}

\section{Introduction}

Human chorionic gonadotropin (hCG) is produced by the placental syncytio-trophoblasts as early as 7-8 blastomere stage of the embryonic development i.e. before actual implantation takes place (M.-L.Bonduelle et al. 1988). The reliable detection of hCG in maternal blood usually coincides with the embryonic implantation phase which is around 7 days post fertilization (Ahmed et al. 1983). While serial hCG levels aren't usually monitored in spontaneous pregnancies, women undergoing assisted reproductive technologies (ART) treatments usually necessitate such an approach especially after embryo transfer (ET). It is believed that hCG levels taken post ET have a diagnostic and prognostic value when it comes to miscarriages, ectopic pregnancies, predicting multiple gestations as well as live births (Schmidt et al., 1994, McCoy et al. 2009).

\section{Discussion}

Despite the lack of consensus on the hCG cutoff values that correlate with the best ART outcomes, a bulk of the studies use a value of $70 \mathrm{mIU} / \mathrm{ml}$ on day 14 post ovum pickup as an acceptable reference value (Sung et al. 2016). Values equivalent to $5 \mathrm{mIU} / \mathrm{mL}$ or below are judged as negative pregnancy tests (Sung et al. 2016, Maslow et al. 2016). It is believed that the amount of hCG produced reflects the mass of the trophoblast tissue as well as it's function (Porat et al. 2007). Despite the discrepancies in the literature, there is a certain agreement that very low initial hCG levels are associated with adverse pregnancy outcomes and intra-uterine growth restriction. This can be explained by the small placental mass with a suboptimal function thus preventing normal fetal growth (Haddad et al. 1999, Krantz et al. 2004, Porat et al. 2007). A possible explanation for the latter might be that some embryos have a division lag, thus a later or abnormal implantation due to variances in trophoblast differentiation (invasive/extravillous versus hCG-producing/ villous phenotype) in an endometrium of decreased receptivity (Bolton et al. 1989, Woodward et al. 1993, Smith et al. 2004, Morse et al. 2016). Jukic et al. found out that smoking status and age at menarche affected the time of implantation by almost 24 hours and thus a late hCG rise. Current active or passive smoking status was significantly associated with delayed implantation. Younger age at menarche (younger than 12 years of age) was also found to be associated with a slow initial hCG rise (Jukic et al.2011). On another note, low initial hCG levels from 1.0 to $5.0 \mathrm{mIU} / \mathrm{mL}$ might be due to a false negative result related to laboratory methodology used for the hCG titration (Maslow et al. 2016). It s worth mentioning that it's not only the initial hCG value but the doubling time as well as the hCGrise curve is more correlated with the pregnancy outcome (Shamonki et al. 2009, Maslow et al. 2016, Morse et al. 2016). A doubling time of 2 days has been set as the best predictor of live birth rate although an increase rate as low as $53 \%$ can also predict a viable pregnancy (Shamonki et al. 2009, Seeber et al. 2012). Initial hCG value post ET is important to diagnose a possible pregnancy, however it doesn't correlate alone with the possibility of a live birth. Serial hCG levels are important especially when the initial values are lower than the cut-off value.

\section{References}

1. Ahmed, A. G., and A. Klopper. "Diagnosis of early pregnancy by assay of placental proteins.” BJOG: An International Journal of Obstetrics \& Gynaecology 90.7 (1983): 604-611.

2. Bolton VN, Hawes SM, Taylor CT, Parsons JH. Development of spare human preimplantation embryos in vitro: an analysis of the correlations among gross morphology, cleavage rates, and development to the blastocyst. $J$ In Vitro Fert Embryo Transf 1989; 6: 30-5

3. Bonduelle, M-L., et al. "Chorionic gonadotrophin- $\beta$ mRNA, a trophoblast marker, is expressed in human 8-cell embryos derived from tripronucleate zygotes." Human Reproduction 3.7 (1988): 909-914. 
4. Haddad, Bassam, et al. "Predictive value of early human chorionic gonadotrophin serum profiles for fetal growth retardation." Human Reproduction 14.11 (1999): $2872-2875$.

5. Jukic, A. M. Z., et al. "The association of maternal factors with delayed implantation and the initial rise of urinary human chorionic gonadotrophin." Human reproduction 26.4 (2011): 920-926.

6. Krantz, David, et al. "Association of extreme first-trimester free human chorionic gonadotropin- $\beta$, pregnancy-associated plasma protein A, and nuchal translucency with intrauterine growth restriction and other adverse pregnancy outcomes.' American Journal of Obstetrics \& Gynecology 191.4 (2004): 1452-1458.

7. Maslow, Bat-Sheva L., et al. "Occult abnormal pregnancies after first post-embryo transfer serum beta-human chorionic gonadotropin levels of 1.0-5.0 mIU/mL." Fertility and sterility 105.4 (2016): 938-945.

8. McCoy, Travis W., Steven T. Nakajima, and Henry CL Bohler. "Age and a single day-14 $\beta$-HCG can predict ongoing pregnancy following IVF." Reproductive biomedicine online 19.1 (2009): 114-120.

9. Morse, Christopher B., et al. "Association of the very early rise of human chorionic gonadotropin with adverse outcomes in singleton pregnancies after in vitro fertilization." Fertility and sterility 105.5 (2016): 1208-1214
10. Porat, Shay, et al. "Early serum $\beta$-human chorionic gonadotropin in pregnancies after in vitro fertilization: contribution of treatment variables and prediction of long-term pregnancy outcome." Fertility and sterility 88.1 (2007): 82-89.

11. Schmidt, Lila L., et al. "The predictive value of a single beta human chorionic gonadotropin in pregnancies achieved by assisted reproductive technology." Fertility and sterility 62.2 (1994): 333-338.

12. Seeber, Beata E. "What serial hCG can tell you, and cannot tell you, about an early pregnancy." Fertility and sterility 98.5 (2012): 1074-1077.

13. Shamonki, Mousa I., et al. "Logarithmic curves depicting initial level and rise of serum beta human chorionic gonadotropin and live delivery outcomes with in vitro fertilization: an analysis of 6021 pregnancies." Fertility and sterility 91.5 (2009): $1760-1764$.

14. Sung, Nayoung, et al. "Serum hCG- $\beta$ levels of postovulatory day 12 and 14 with the sequential application of hCG- $\beta$ fold change significantly increased predictability of pregnancy outcome after IVF-ET cycle." Journal of assisted reproduction and genetics 33.9 (2016): 1185-1194.

15. Woodward BJ, Lenton EA, Turner K. Human chorionic gonadotrophin: embryonic secretion is a time-dependent phenomenon. Hum Reprod 1993;8: 1463-8.

Citation:

Mohammad Jaffa, Layal Hamdar, Anastasia Salame (2019) Late Rise Human Chorionic Gonadotropin after Embryo Transfer: Causality and Significance! A mini review. Integr Gyn Obstet $J$ Volume 2(3): 1-2. 